\title{
Evaluation of Orthogonal Polynomials and Relationship to Evaluating Multiple Integrals
}

\author{
By P. M. Hirsch
}

1. Introduction. Orthogonal polynomials and their relationship to numerical integration formulas in one variable are well known (see [7], for example), but only a few [1], [8]-[10] have attempted to extend these results to orthogonal polynomials in several variables and multiple integrals.

This paper will look at properties and methods for calculating orthogonal polynomials in several variables, also showing the relationship of orthogonal polynomials to evaluate multiple integrals. In order to discuss polynomials in several variables it will be necessary to give a linear ordering for the monomials of a polynomial. Several orderings are possible, the following having been stated by $\mathrm{M}$. Weisfeld [12].

Let $J$ be the set of $n$-tuples of nonnegative integers and let $\Phi$ be the corresponding set of monomials, that is $j=\left(j_{1}, j_{2}, \cdots, j_{n}\right) \in J$ and $\phi_{j}=$ $x_{1}{ }^{j_{1}} x_{2}{ }^{j_{2}} \cdots x_{n}{ }^{j_{n}} \in \Phi$. Define $\sigma_{k}(j)=\sum_{i=k}^{n} j_{i}$ and order $J$ as follows: if $i, j \in J$, then $i<j$ iff $\sigma_{k}(i)=\sigma_{k}(j), k=1,2, \cdots, r-1$ and $\sigma_{r}(i)<\sigma_{r}(j)$ for some $r, 1 \leqq r \leqq n$. This ordering on $J$ induces a linear ordering on $\Phi$.

For example, if we have two variables $x_{1}$ and $x_{2}$, the ordering would be 1 , $x_{1}, x_{2}, x_{1}^{2}, x_{1} x_{2}, x_{2}{ }^{2}, x_{1}^{3}, x_{1}^{2} x_{2}, x_{1} x_{2}{ }^{2}, x_{2}{ }^{3}, \cdots, x_{1}{ }^{n}, x_{1}{ }^{n-1} x_{2}, x_{1}{ }^{n-2} x_{2}{ }^{2}, \cdots, x_{2}{ }^{n}$.

We may now express a polynomial in $n$-variables as

$$
P(X)=\sum_{\sigma(i)=0}^{m} A_{i} \phi_{i}(X) \quad \text { where } \sigma(i)=\sigma_{1}(i) .
$$

Definition. A polynomial in $n$-variables is of degree $m$ if the coefficient $A_{i} \neq 0$ for some $n$-tuple $i$ such that $\sigma(i)=m$. Note that a polynomial of degree $m$ in $n$ variables has at most $C_{m+n, n}$ monomials.

In order to define orthogonal polynomials one must first make the following definitions.

Definition. Given a domain $D \subset E^{n}$ with positive outer measure and a weight function, $W(X)$ of constant sign on $D$, then for functions $f(X)$ and $g(X)$ such that $W(X) f(X) g(X)$ is integrable over $D$, we can define the scalar product operator as

$$
(f, g)=\int \underset{D}{\ldots} \int W(X) f(X) g(X) d X .
$$

It will be assumed that the domain $D$ and the weight function $W(X)$ are chosen so that the scalar product operator is defined for polynomials.

Definition. Two functions $f(X)$ and $g(X)$ are orthogonal if $(f, g)=0$.

Definition. An orthogonal polynomial is a polynomial that is orthogonal to all polynomials of lower degree.

In the one-variable case an orthogonal polynomial of degree $m$ was unique up

Received July 14, 1967. Revised September 5, 1967. 
to a multiplicative constant. In more than one variable there are many linearly independent orthogonal polynomials of degree $m$. The next theorem gives a basis set for the orthogonal polynomials of degree $m$.

Theorem 1. Let $\mathfrak{P}_{m}$ be the set of all orthogonal polynomials of degree $m$, then $\mathfrak{P}_{m}$ has dimension $C_{n+m-1, n-1}$ and has a basis of the form

$$
B_{m}=\left\{b_{j}: b_{j}=\phi_{j}(X)+\sum_{\sigma(i)=0}^{m-1} A_{j, i} \phi_{i}(X), \sigma(j)=m\right\} .
$$

Proof. First it will be necessary to show that a subset $B_{m}$ of $\mathfrak{P}_{m}$ exists of the form described in the theorem. Since each element $b_{j}$ of $B_{m}$ should be an orthogonal polynomial, they must satisfy the $C_{m-1+n, n}$ equations

$$
\left(b_{j}, \phi_{i}\right)=\left(\phi_{j}, \phi_{i}\right)+\sum_{\sigma(l)=0}^{m-1} A_{j, l}\left(\phi_{l}, \phi_{i}\right)=0 \quad \text { where } \sigma(i)=0,1, \cdots, m-1 .
$$

A solution will exist provided the Gram determinant $\left|\left(\phi_{i}, \phi_{1}\right) \cdots\left(\phi_{i}, \phi_{k}\right)\right|$, $i=1, \cdots, k \neq 0$ where $k=C_{m-1+n, n}$. According to Davis [3] the Gram determinant is not zero if $\phi_{1}, \cdots, \phi_{k}$ are linearly independent. Since $\phi_{1}, \phi_{2}, \cdots, \phi_{k}$ are the linearly independent monomials, we have that a subset $B_{m}$, as described in the theorem, of $\mathfrak{B}_{m}$ exists.

Let us now show that $B_{n}$ is a generating set of $\mathfrak{P}_{m}$. Let $P_{m}(X) \in \mathfrak{P}_{m}$ then

$$
P_{m}(X)=\sum_{\sigma(i)=0}^{m-1} C_{i} \phi_{i}(X)+\sum_{\sigma(j)=m} C_{j} \phi_{j}(X) .
$$

If $Q_{m-1}(X)=P_{m}(X)-\sum_{\sigma(j)=m} C_{j} b_{j}$, then $Q_{m-1}(X)$ is a polynomial of degree at most $m-1$. Therefore we have that

$$
\left(Q_{m-1}, Q_{m-1}\right)=\left(P_{m}, Q_{m-1}\right)-\sum_{\sigma(j)=m} C_{j}\left(b_{j}, Q_{m-1}\right)=0 .
$$

Then $Q_{m-1}=0$ and $P_{m}(X)=\sum_{\sigma(j)=k} C_{j} b_{j}$. Therefore $B_{m}$ generates all the elements for $\mathfrak{B}_{m}$.

One can also see that if one omits any element, $b_{j}$, from the set $B_{m}$, the remaining set is not a generating set since one could not generate $b_{j}$ from the remaining elements at $B_{m}$. Therefore $B_{m}$ is a basis set.

The order at $B_{m}$ is $C_{n+m-1, n-1}$ and this is therefore also the dimension of the space $\mathfrak{B}_{m} .:$ :

2. Generation. There are several distinct methods for generating a basis set for orthogonal polynomials in several variables. These methods follow closely similar methods for generating the one-variable orthogonal polynomials.

The first method follows from the proof of Theorem 1 . The $C_{m+n-1, n-1}$ basis elements for orthogonal polynomials of degree $m$ are of the form:

$$
b_{j}=\phi_{j}(X)+\sum_{\sigma(i)=0}^{m-1} A_{j, i} \phi_{i}(X) \text { where } \sigma(j)=k .
$$

The set of coefficients $\left\{A_{j, i}\right\}$ are found by solving the $C_{m+n-1, n}$ linear equations

$$
0=\left(b_{j}, \phi_{i}\right)=\sum_{\sigma(k)=0}^{m-1} A_{j, k}\left(\phi_{k}, \phi_{i}\right)+\left(\phi_{j}, \phi_{i}\right) \quad \text { where } i=1, \cdots, C_{m+n-1, n} .
$$


This would be the matrix approach to generating the basis set and the resulting basis set is the one mentioned in Theorem 1 .

The second method is to orthogonalize the monomials $\phi_{1}, \phi_{2}, \cdots, \phi_{k-1}, \phi_{k}, \phi_{j}$, where $k=C_{m-1+n, n}$ and $\sigma(j)=m$. Using the Gram-Schmidt method, the basic equations are:

$$
\begin{aligned}
& Q_{1}=\phi_{1} \\
& D_{1}=\left(Q_{1}, Q_{1}\right)^{1 / 2} \\
& P_{1}=Q_{1} / D_{1} \\
& \vdots \\
& Q_{l}=\phi_{l}-\sum_{i=1}^{l-1}\left(\phi_{l}, P_{i}\right) P_{i} \\
& D_{l}=\left(Q_{l}, Q_{l}\right)^{1 / 2} \quad \text { for } \quad l=2,3, \cdots, k=C_{m-1+n, n} . \\
& P_{l}=Q_{l} / D_{l}
\end{aligned}
$$

Then the basis set for $\mathfrak{B}_{m}$ is

$$
B_{m}=\left\{b_{j}: b_{j}=\phi_{j}-\sum_{i=1}^{k}\left(\phi_{j}, P_{i}\right) P_{i}, \sigma(j)=m\right\} .
$$

The third method is to find a partial differential equation with boundary condition such that the $m$ th partial derivative of the solution is related to a basis element of the orthogonal polynomials of degree $m$. The method is similar to one suggested by Gröbner [4].

Let $P_{j}(X)$ be an $m$ th-degree orthogonal polynomial over a region $D$ with a positive weight function $W(X)$. Let $U_{j}(X)$ be a function such that

$$
\frac{\partial^{m} u_{j}(X)}{\partial^{a_{1}} x_{1} \cdots \partial^{a_{n}} \cdot x_{n}}=W(X) P(X), \text { where } \sum_{i=1}^{n} a_{i}=m .
$$

Since $P_{j}(X)$ is an $m$ th degree polynomial, we have that

$$
\frac{\partial^{m+1}}{\partial^{a_{1}+1} x_{1} \cdots \partial^{a_{n}} \cdot x_{n}} \frac{1}{W(X)}\left(\frac{\partial^{m} U_{j}(X)}{\partial^{a_{1}} x_{1} \cdots \partial^{a_{n}} x_{n}}\right)=0 .
$$

To get the boundary conditions to the partial differential equation one must integrate by parts. Using a generalization of Green's theorem to $n$ dimensions one has

$$
\begin{aligned}
\iint{ }_{D} \iint_{j}(X) \frac{\partial V(X)}{\partial x_{i}} d X= & \pm \int \underset{\partial D}{\iint} U(X) V(X) \cos \left(n, x_{i}\right) d S \\
& -\iint \ldots \int V(X) \frac{\partial U(X)}{\partial x_{i}} d X
\end{aligned}
$$

where the plus or minus sign depends on the orientation of $\partial D$ and on the coordinate $x_{i}$.

Since $P_{j}(X)$ is an orthogonal polynomial, if $P_{m-1}$ is any polynomial of degree at most $m-1$ then

$$
0=\iint \ldots \int_{D} \frac{\partial^{m} U_{j}(X)}{\partial^{a_{1}} x_{1} \cdots \partial^{a_{n}} x_{n}} P_{m-1}(X) d X .
$$


Using the previous equation to integrate by parts the above equation one can reduce the order of differentiation on $U_{j}(X)$ while increasing the order of differentiation on $P_{m-1}(X)$. There are many ways of accomplishing this but after $m-1$ steps one gets the $m$ th partial of $P_{m-1}(X)$ which is zero. Combining all these ways by summation we have

$$
\begin{aligned}
0= & \pm \sum_{u_{1}+\cdots+u_{n}=0 ;}^{n-1} \sum_{0 \leqq u_{i} \leqq a_{i}} \sum_{k=1}^{n} \int \ldots \int \frac{\partial^{m-\left(u_{1}+\cdots+u_{n}\right)-1} U_{j}(X)}{\partial^{a_{1}-u_{1}} x_{1} \cdots \partial^{a_{k}-u_{k}-1} x_{k} \cdots \partial^{a_{n}-u_{n}} \cdot x_{n}} \\
& \cdot \frac{\partial^{u_{1}+\cdots+u_{n}} P_{j}(X)}{\partial x_{1}{ }^{u_{1}} \cdots \partial x_{n}{ }^{u_{n}}} \cos (n, x) d S .
\end{aligned}
$$

The polynomial $P_{m-1}$ was an arbitrary polynomial of degree at most $m-1$, therefore the only way the above equation can be valid is that either $\cos \left(n, x_{k}\right)=0$ or the respective partials of $U_{j}(X)$ are zero; this gives us the following boundary conditions to the partial differential equation:

$$
\begin{array}{cl} 
& \text { if } v_{1}<a_{i} \text { for all points on } \partial D, i=1, \cdots, n, \\
\frac{\partial^{m} U_{j}(X)}{\partial^{v_{1}} x_{1} \cdots \partial^{v_{n}} x_{n}}=0 & \begin{array}{l}
\text { if } v_{i}=a_{i} \text { for all points on } \partial D, \\
\text { if } v_{j}<a_{j} \text { where } \cos \left(n, x_{i}\right) \neq 0 \text { for each } i=1, \cdots, n \\
\text { and every } j=1, \cdots, i-1, i+1, \cdots, n .
\end{array}
\end{array}
$$

Note that the differential equation with its boundary conditions produces a basis set which is different from the basis produced by the first two methods.

3. Numerical Integration. In this section we will show the relationship of orthogonal polynomials in several variables to the evaluation of multiple integrals.

Given an integer $m$, a region $D \subset E^{n}$ with positive outer measure and an integrable weight function $W(X)$ of constant sign on $D$ whose integral, over any region $D^{\prime} \subset D$ with positive outer measure, is positive: the problem is to find points $X_{1}, \cdots, X_{r}$ and weights $u_{i}, \cdots, u_{r}$ so that the equation

$$
I(f)=\int \underset{D}{\ldots} \int W(X) f(X) d X=\sum_{i=1}^{r} u_{i} f\left(X_{i}\right)
$$

holds whenever $f(X)$ is a polynomial of degree at most $m$.

Goals that one would like to achieve are that the points $X_{1}, X_{2}, \cdots, X_{r}$ are real, in the region $D$ and that $r$ is a minimum. Vladimir Tchakaloff [11] showed that if the weights $u_{1}, u_{2}, \cdots, u_{r}$ are to be positive, then in general $C_{m+n, n}$ points are needed. In this paper we will look at regions and integration formulas that require fewer than $C_{m+n, n}$ points and still meet most or all of the above goals.

A polynomial of degree $m$ in $n$-variables has $C_{m+n, n}$ monomials. Replacing $f(X)$ in the above equation by each of the monomials, one gets $C_{m+n, n}$ nonlinear equations in terms of the $(n+1) r$ unknowns $X_{i}=\left(x_{1_{i}}, x_{2_{i}}, \cdots, x_{n_{i}}\right) u_{i}, i=1, \cdots, r$. If the number of equations is to equal the number of unknowns one has $r=C_{m+n, n} /(n+1)$. There are however some integration formulas that use fewer than this number of points [5].

In this section it will be shown that if an integration formula of precision $m$ has less than $C_{m+n, n}$ points the points of this integration formula will be zeros of a polynomial which is orthogonal to a set of polynomials of lower degree. 
Theorem 2. If an integration formula of precision $m$ has $r<C_{m+n, n}$ points then these $r$ points are zeros of a polynomial of degree $k, 0<k \leqq m$.

Proof. Let $r$ be the number of points in the integration formula and let $j$, $1 \leqq j<C_{m+n, n}$, be the rank of the matrix $\left|\phi_{1}\left(X_{i}\right) \cdots \phi_{r}\left(X_{i}\right)\right|, i=1, \cdots, r$. For some subsets $\left\{k_{1}, \cdots, k_{j}\right\},\left\{i_{1}, \cdots, i_{j}\right\}$ of $\{1, \cdots, r\}$ we have the determinant $\left|\phi_{k_{1}}\left(X_{i}\right) \cdots \phi_{k_{j}}\left(X_{i}\right)\right|, i=i_{1}, \cdots, i_{j} \neq 0$. Therefore the polynomial

$$
P(X)=\left|\phi_{k_{1}}\left(X_{i}\right) \cdots \phi_{k_{j+1}}\left(X_{i}\right)\right|, \quad i=*, i_{1}, \cdots, i_{j},
$$

(the asterisk implies that in the first row the symbol $X_{i}$ is replaced by the variable $X$ ) has all the points of the integration formula as zeros and is of degree at most $m$, and not identically zero. ::

LEmma. If $P(X)$ is a polynomial that is not identically zero then the set of zeros of $P(X)$ is of measure zero.

Proof. Assume that a polynomial $P(X)$ is zero on a set $Z$ which has positive measure. Place all the variables except one equal to some constant $c=$ $\left(c_{1}, c_{2}, \cdots, c_{n-1}\right)$, then we can write $P(X)=Q_{c}\left(X_{n}\right)$.

If $l_{n} \cap Z$ is not empty for a line $l$ parallel to one of the axes, in this case the $x_{n}$ axis, then almost always the set $l \cap Z$ has positive measure [5]. Therefore for some constant $c, Q_{c}\left(X_{n}\right)=0$ has more than a finite number of roots. By the fundamental theorem of algebra this implies $Q_{c}\left(X_{n}\right)=0$. The coefficients of $Q_{c}\left(X_{n}\right)$ are polynomials in the remaining $n-1$ variables and by induction we have that $P(X) \equiv 0$. ::

Theorem 3. An integration formula in $E^{n}$ of precision $m$ must have more than $C_{[m / 2]+n, n}$ points.

Proof. If an integration formula in $E^{n}$ of precision $m$ has not more than $C_{[m / 2]+n, n}$ points, then the polynomial of Theorem 2 is of degree at most $[\mathrm{m} / 2]$, and $P^{2}(X)$ has degree at most $m$. Since the integration formula is of precision $m$ we have that $\iint_{D} W(X) P^{2}(X) d X=0$. We were given that $\iint_{D} W(X)>0$ and $W(X) \geqq 0$ on $D$; therefore for some $D^{\prime} \subset D, \mu\left(D^{\prime}\right)>0$ we have that $W(X)>0$ on $D^{\prime}$. By the lemma $P^{2}(X)>0$ on $\tilde{D}=D^{\prime}-E$ where $\mu(E)=0$. Therefore $W(X) P^{2}(X)>0$ on $\tilde{D}$ and $\iint_{D} W(X) P^{2}(X) d X>\iint_{\tilde{D}} W(X) P^{2}(X) d X>0$ which is a contradiction. ::

Theorem 4. The polynomial $P(X)$ of degree $k$ which has the points of the integration formula of precision $m$ as roots is orthogonal to all polynomials of degree less than or equal to $m-k$.

Proof. Let $Q(X)$ be any polynomial of degree less than or equal to $m-k$. The integration formula of precision $m$ is exact for polynomials of degree less than $m$; hence $(P, Q)=\iint_{D} W(X) P(X) Q(X) d X=0$. Therefore $P(X)$ is orthogonal to polynomials of degree at most $m-k$.::

IBM Scientific Center

6900 Fanning Street

Houston, Texas 77025

1. P. Appel, "Sur une classe de polynômes a deux variables et le calcul approché des intégrales doubles," Ann. Fac. Sci. Univ. Toulouse, v. 4, 1890, pp. H.1-H.20.

2. A. ERdelyi et AL., Higher Transcendental Functions, Vol. 2, Bateman Manuscript Project, McGraw-Hill, New York, 1953. MR 15, 419. 
3. P. J. Davis, Interpolation and Approximation, Blaisdell, New York, 1963. MR 28 \#393.

4. Wolfgang Gröbner, "Über die Konstruktion von Systemen orthogonaler Polynome in ein- und zwei-dimensionalen Bereichen," Monatsh. Math., v. 52, 1948, pp. 38-54. MR 9, 430.

5. P. R. Halmos, Measure Theory, Van Nostrand, New York, 1964. MR 11, 504.

6. P. C. HAMmer, "Numerical evaluation of multiple integrals," On Numerical Approximation, Univ. of Wisconsin Press, Madison, Wis., 1959, pp. 99-115. MR 20 \#6788.

7. F. B. Hildebrand, Introduction to Numerical Analysis, McGraw-Hill, New York, 1956. MR 17, 788 .

8. P. M. Hinsch, Numerical Evaluation and Estimation of Multiple Integrals, Ph.D. Thesis, Univ. of Wisconsin, Madison, Wis., 1966. (Unpublished.)

9. Johann Radon, "Zur mechanischen Kubatur," Monatsh. Math. Phys., v. 52, 1948, pp. 286-300. MR 11, 405.

10. A. H. STRoud, "Integration formulas and orthogonal polynomials," SIAM J. Numer. Anal., v. 4, 1967, pp. 381-389.

11. Vladimir TCHakaloff, "Formulas of mechanical cubature with non-negative coefficients," Bull. Sci. Math., (2), v. 81, 1957, pp. 123-134. MR 20 \#1145.

12. MoRRIS WEISFELD, "Orthogonal polynomials in several variables," Numer. Math., v. 1, 1959, pp. 38-40. MR 21 \#1687. 